\title{
Mechanisms of void shrinkage in aluminium Supplementary Material
}

\author{
Zezhong Zhang, ${ }^{a}$ Tianyu Liu,${ }^{a}$ Andrew E. Smith, ${ }^{b}$ Nikhil V. Medhekar, ${ }^{a *}$ \\ Philip N. H. NAKAshima ${ }^{a *}$ AND LAURe Bourgeois ${ }^{a, c *}$ \\ ${ }^{a}$ Department of Materials Science and Engineering, Monash University, Victoria \\ 3800, Australia, ${ }^{b}$ School of Physics and Astronomy, Monash University, Victoria \\ 3800, Australia, and ${ }^{c}$ Monash Centre for Electron Microscopy, Monash University, \\ Victoria 3800, Australia.E-mail: nikhil.medhekar@monash.edu, \\ philip.nakashima@monash.edu,laure.bourgeois@monash.edu
}

Nanovoid, Transmission Electron Microscopy, Vacancies, Diffusion, Aluminium

PREPRINT: Journal of Applied Crystallography A Journal of the International Union of Crystallography 


\section{Additional Transmission Electron Microscopy Evidence}
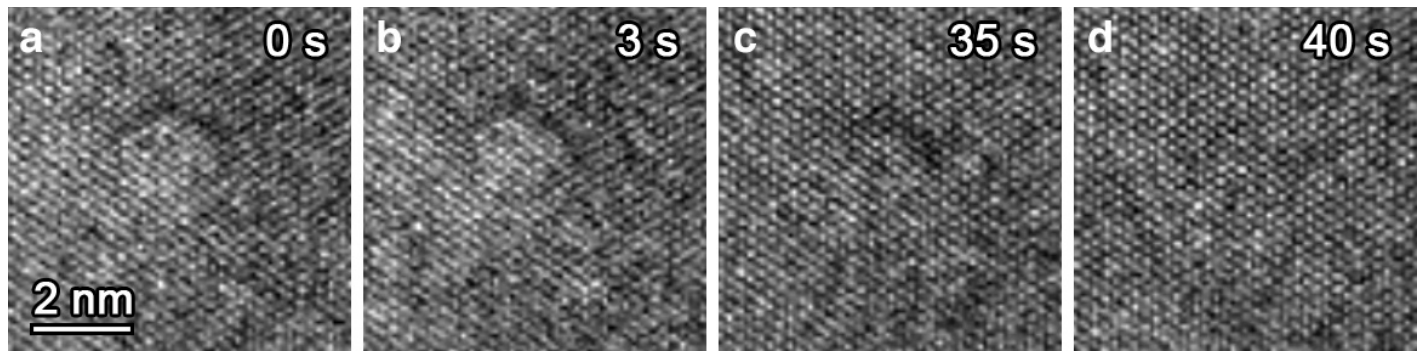

Fig. S1. The last moments of a void's life at $100^{\circ} \mathrm{C}$, as imaged in an in-situ annealing TEM experiment under a $200 \mathrm{keV}$ electron beam. The void remains visible until it is $\approx 1 \mathrm{~nm}$ in diameter in $(\mathrm{c})$.
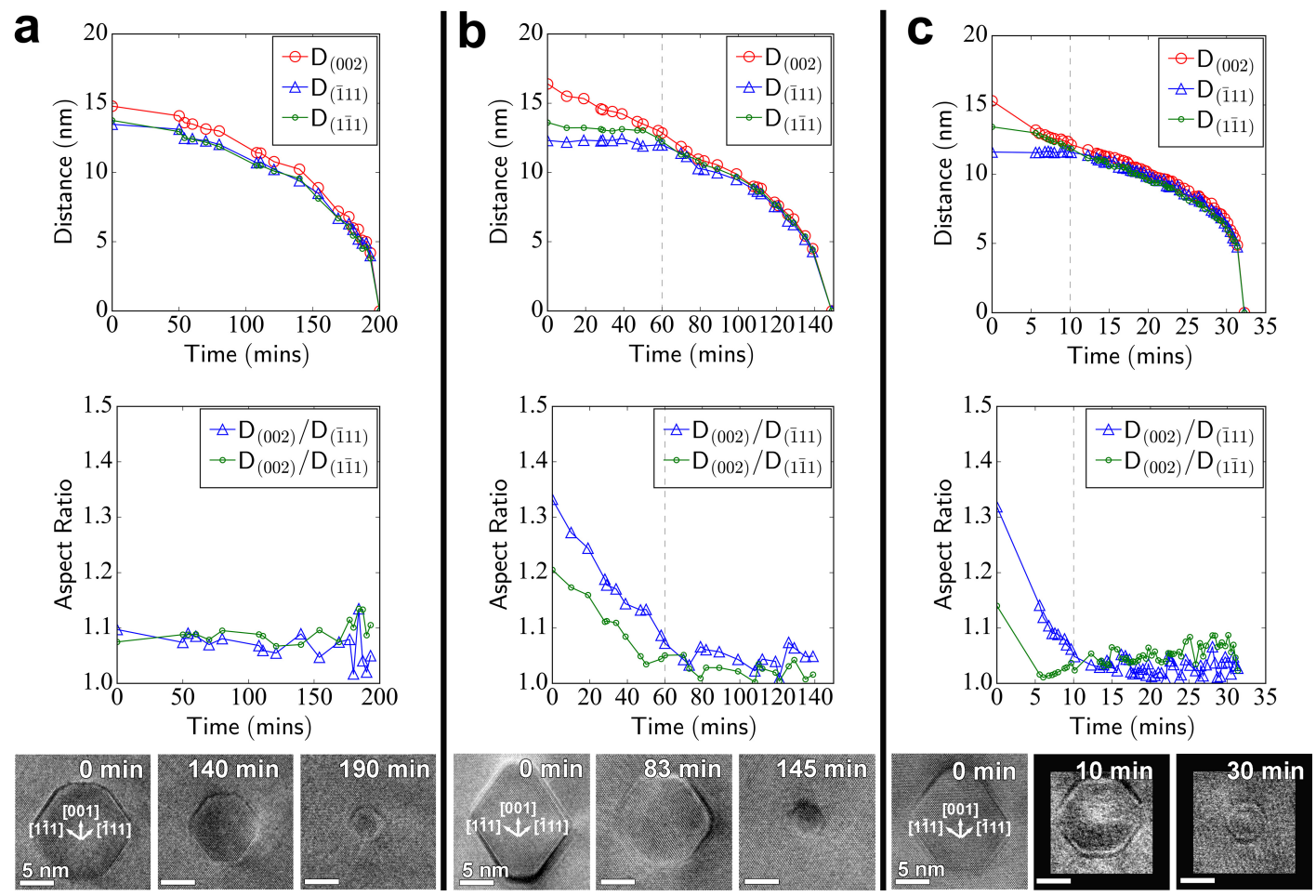

Fig. S2. Void evolution during annealing at (a) $20^{\circ} \mathrm{C}$, (b) $50^{\circ} \mathrm{C}$, and (c) $150^{\circ} \mathrm{C}$ under a $200 \mathrm{keV}$ electron beam. The dashed lines indicate the separation of the two stages. The two-stage behaviour and equilibrium shape are consistent throughout these temperatures. For $20^{\circ} \mathrm{C}$, the void already exhibits a shape at near the equilibrium aspect ratio before annealing starts. For the $150^{\circ} \mathrm{C}$ experiment, the boxed images are snapshots from Supplementary Movie 2. 


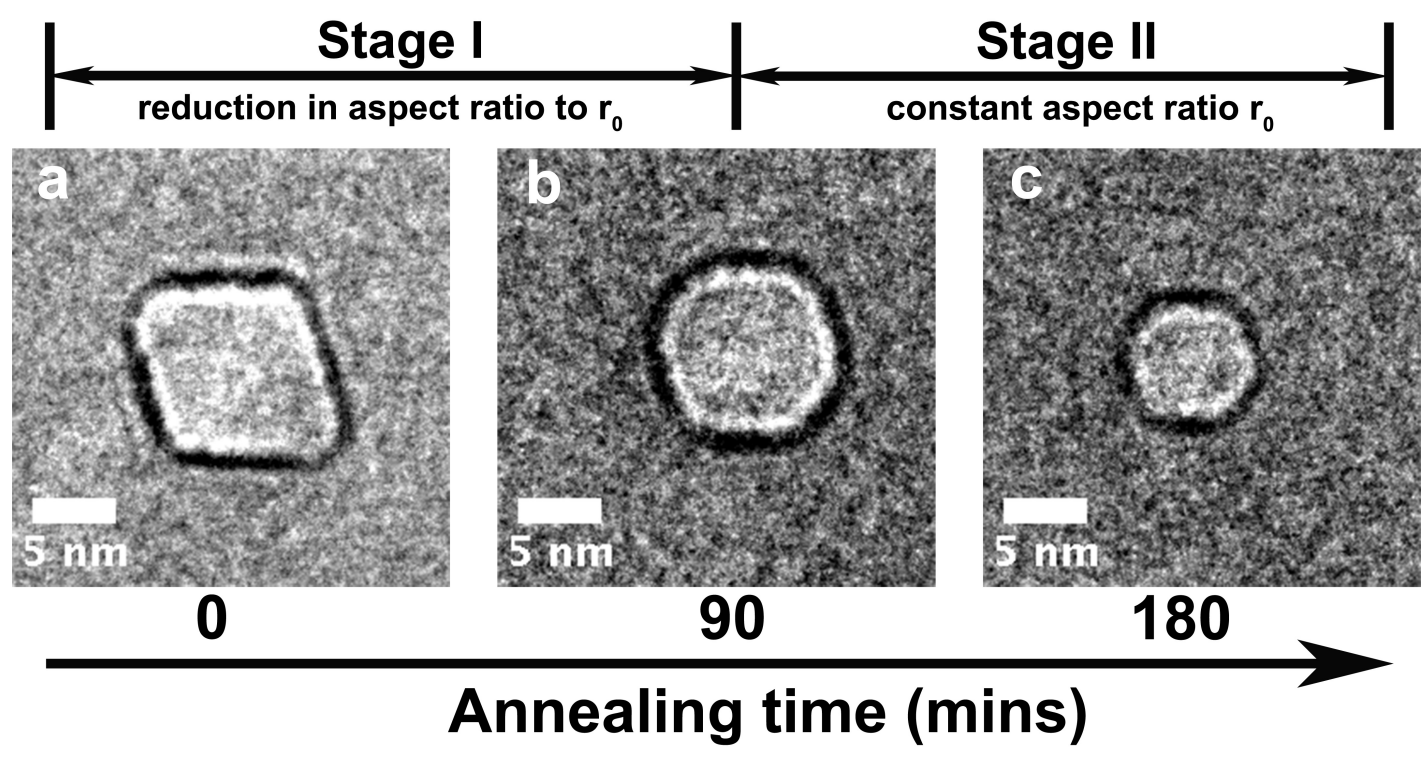

Fig. S3. A void annealed at $130^{\circ} \mathrm{C}$ with the $200 \mathrm{keV}$ electron beam mostly off also exhibits a two-stage shrinkage behaviour, as in the in-situ TEM annealing with the beam continuously on. To minimise the influence from electron irradiation, images were acquired at intervals of $30 \mathrm{~min}$ at low magnification with the beam on for less than $10 \mathrm{~s}$. $(\mathrm{a}-\mathrm{b})$ show the reduction in aspect ratio to $\mathrm{r}_{0} \approx 1.1$, and $(\mathrm{b}-\mathrm{c})$ show $\mathrm{a}$ reduction in size with a constant aspect ratio $\left(\mathrm{r}_{0} \approx 1.1\right)$. 

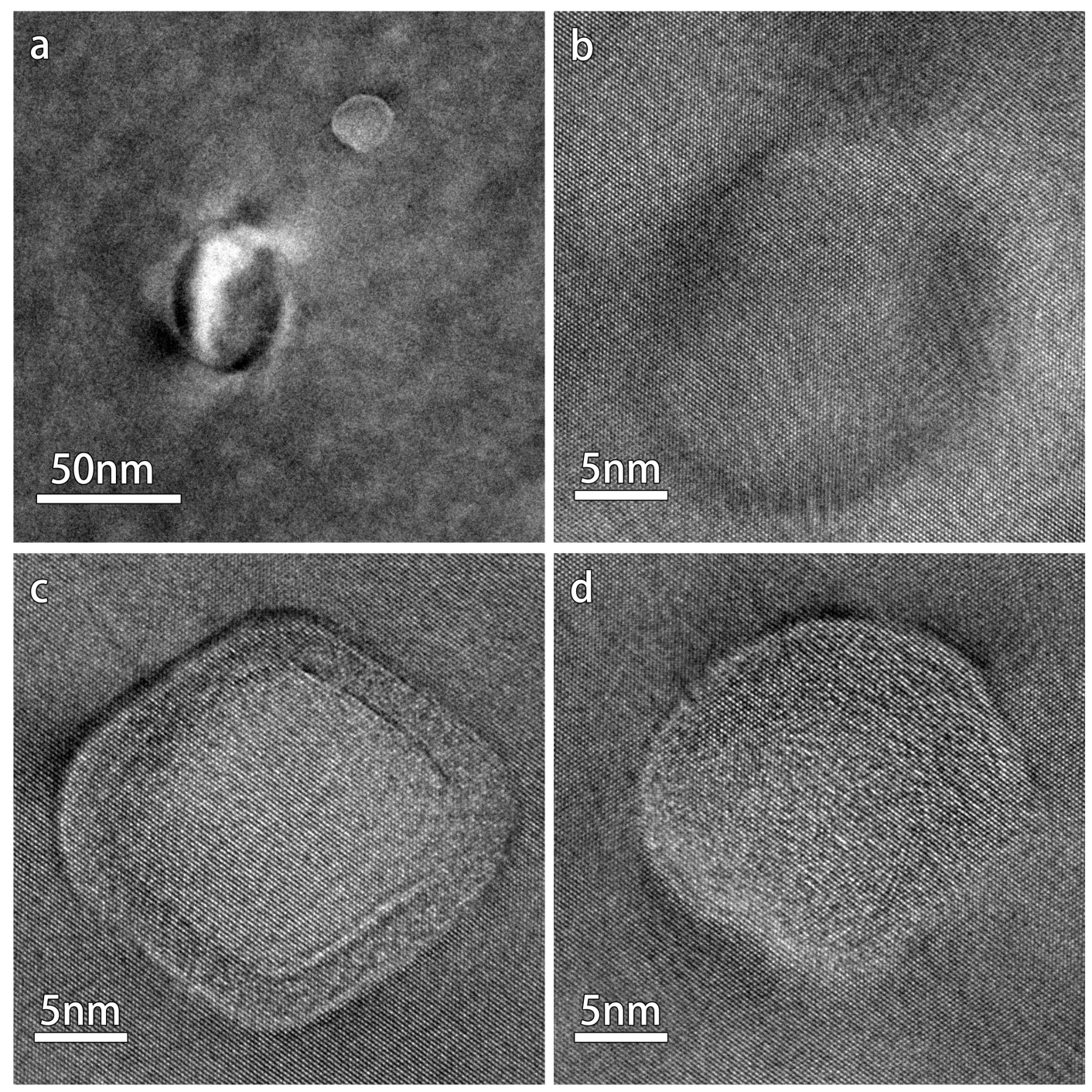

Fig. S4. (a) TEM image showing the region of Fig. 1(a) in the main text, which initially contained two voids, at the end of the in-situ annealing experiment at $100^{\circ}$ C. (b) The lower void has collapsed into a dislocation loop. (c) High-resolution TEM image of the upper void, which exhibits a thick shell before annealing, and (d) is still present after annealing. 

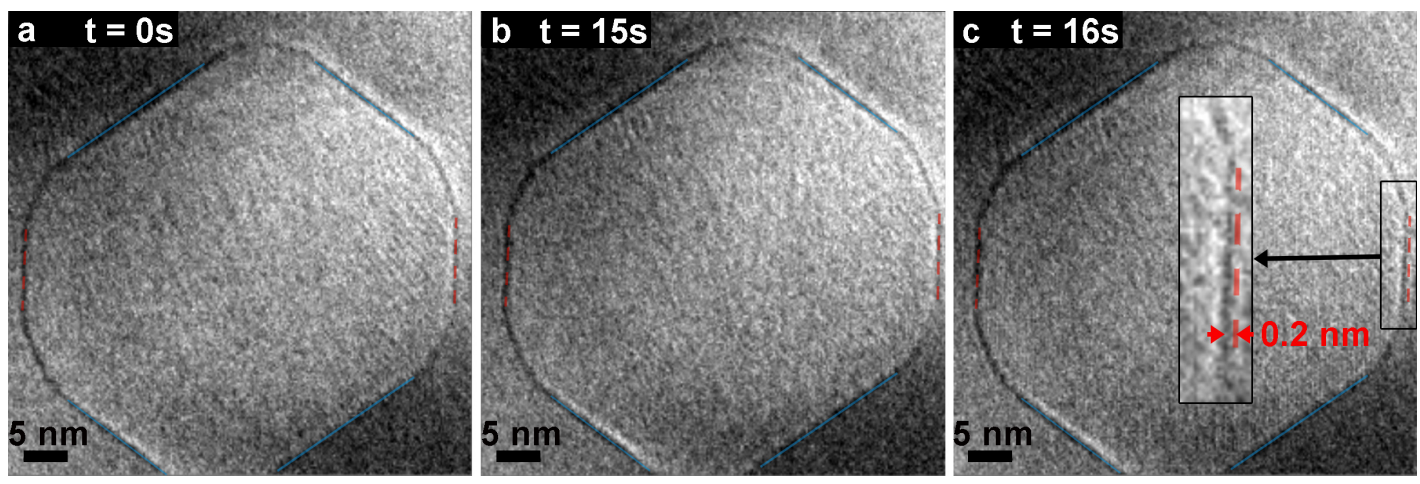

Fig. S5. Snapshots during TEM annealing (at $100^{\circ} \mathrm{C}$ under a $200 \mathrm{keV}$ electron beam) showing that shrinkage takes place one monolayer at a time. The blue lines indicate $\{111\}$ facets edge on, which do not move in the present sequence, and the red lines the $\{002\}$ facets, which move towards the centre of the void in increments of 0.2 nm. 

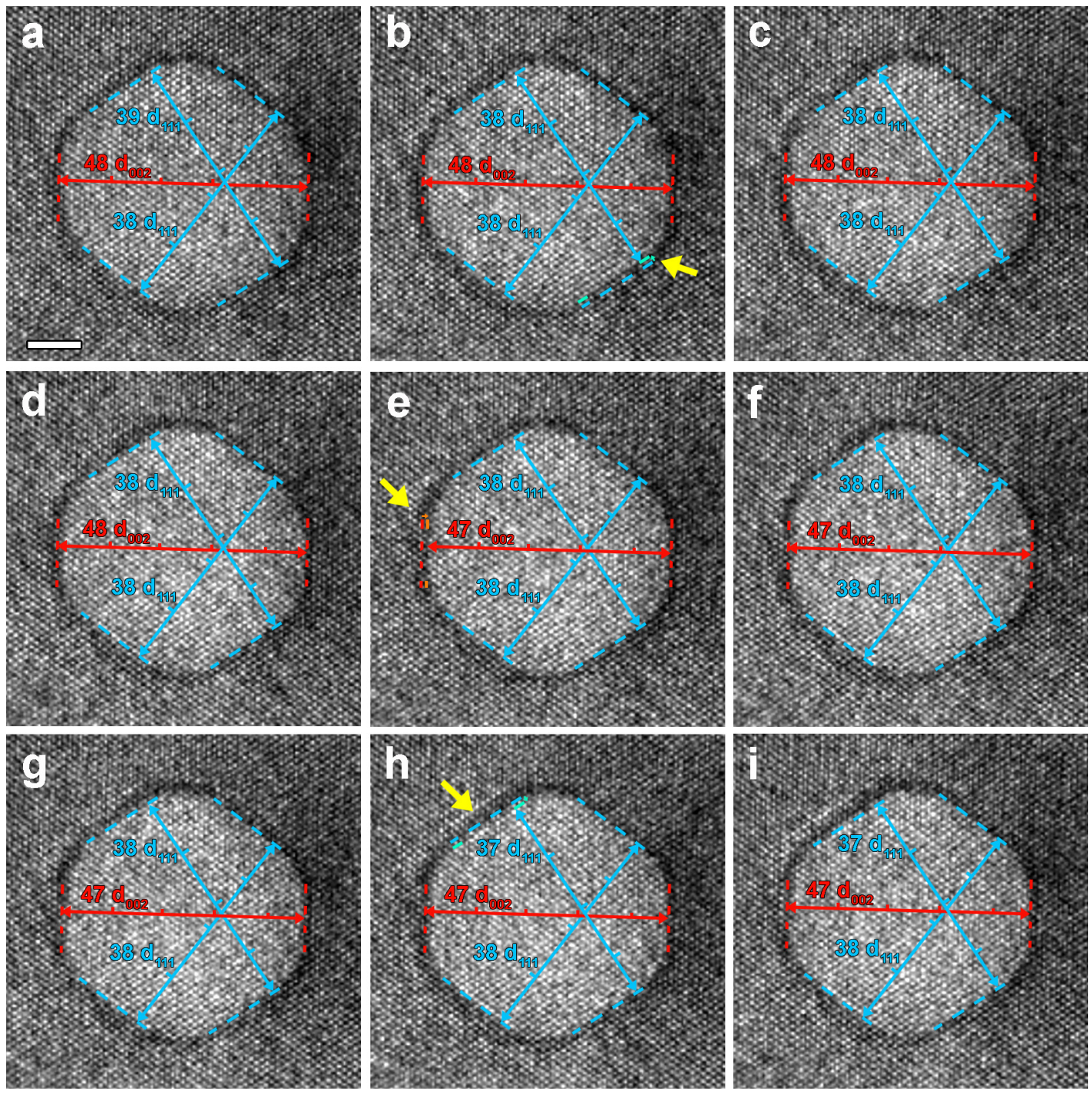

Fig. S6. Three sets of three consecutive snapshots during TEM annealing (at $100^{\circ} \mathrm{C}$ under a $200 \mathrm{keV}$ electron beam) showing that shrinkage takes place one monolayer at a time. The blue lines indicate $\{111\}$ facets edge on and the red lines the $\{002\}$ facets. (a-c) are the components of Fig. 5 in the main text. (d-f) show the reduction of the void size by one $\{002\}$ layer, and (g-i) by one $\{111\}$ layer. 

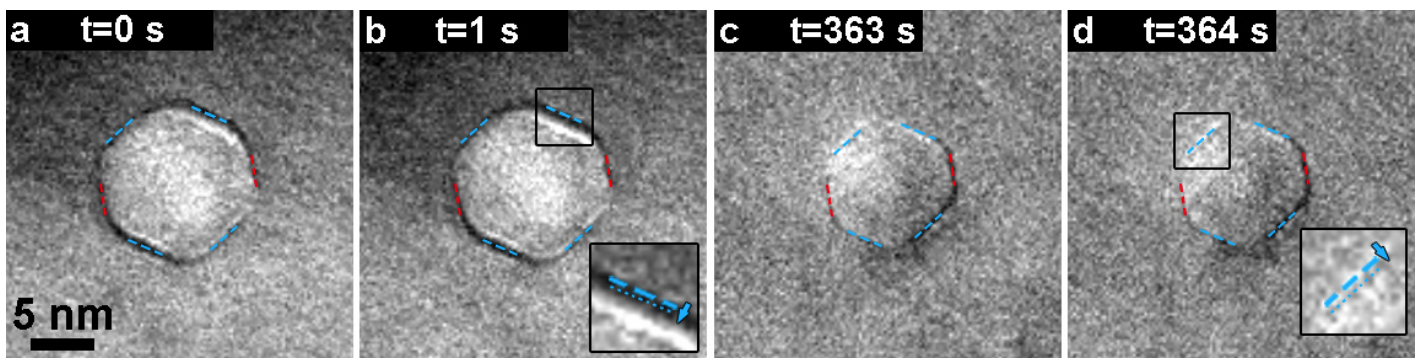

Fig. S7. Void shrinkage under a $160 \mathrm{keV}$ electron beam. It involves single atomic layers for individual facets, as for $200 \mathrm{keV}$. 


\section{Derivation of Equations Describing the Void Geometry}

\subsection{Truncated Octahedron in the Continuum Model}

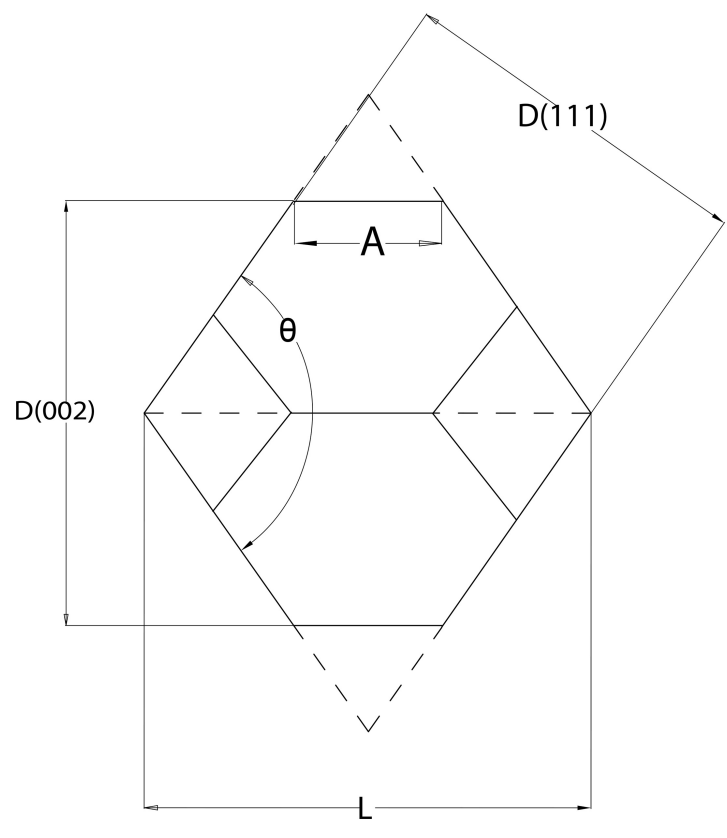

(a)

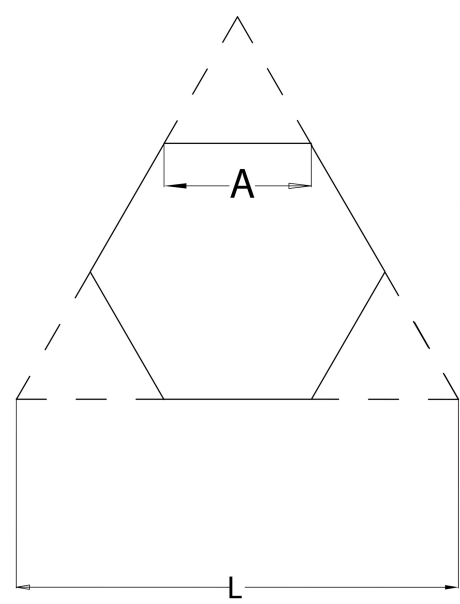

(b)

Fig. S8. (a) Schematic diagram of a void viewed in a $<110>$ direction. The continuous line outlines the truncated octahedron, and the dashed line the pyramidal truncations. All parameters are defined in the text. (b) One hexagonal $\{111\}$ facet of an $\{002\}$-truncated octahedron viewed in a $<111>$ direction.

The angle $\theta$ between two facets $\left(h_{1} k_{1} l_{1}\right)$ and $\left(h_{2} k_{2} l_{2}\right)$ is

$$
\cos \theta=\frac{h_{1} h_{2}+k_{1} k_{2}+l_{1} l_{2}}{\sqrt{h_{1}^{2}+k_{1}^{2}+l_{1}^{2}} \sqrt{h_{2}^{2}+k_{2}^{2}+l_{2}^{2}}} .
$$

Accordingly, the angle $\theta$ in Fig. S8(a) between two intersecting $\{111\}$ facets is

$$
\theta=\arccos \left(-\frac{1}{3}\right)
$$

IUCr macros version 2.1.6: 2014/01/16 
The side length of the untruncated octahedron $L$ is

$$
\begin{aligned}
L & =D_{\{111\}} / \sin \left(\frac{1}{2} \theta\right) \\
& =\frac{\sqrt{3}}{\sqrt{2}} D_{\{111\}} .
\end{aligned}
$$

Since the height of the octahedron is $\sqrt{2} L$, by similar triangles, the side length $A$ of the $\{002\}$ facets is

$$
\begin{aligned}
A & =\frac{\sqrt{2}}{2}\left(\sqrt{2} L-D_{\{002\}}\right) \\
& =\frac{\sqrt{2}}{2}\left(\sqrt{3} D_{\{111\}}-D_{\{002\}}\right) .
\end{aligned}
$$

We define the aspect ratio $r$ as

$$
r=D_{\{002\}} / D_{\{111\}}
$$

Note that the aspect ratio is $r=1.16$ for an Archimedean truncated octahedron, where truncations are taken at one third of the regular octahedron side length.

The surface area $S_{\{002\}}$ of the six square $\{002\}$ facets, each with surface area $S_{(002)}$, is then

$$
\begin{aligned}
S_{\{002\}} & =6 S_{(002)} \\
& =6 A^{2} \\
& =3(\sqrt{3}-r)^{2} D_{\{111\}}^{2} .
\end{aligned}
$$

The surface area $S_{\{111\}}$ for the eight hexagonal $\{111\}$ facets, each with surface area $S_{(111)}$ shown in Fig. S8(b), is then

$$
\begin{aligned}
S_{\{111\}} & =8 S_{(111)} \\
& =8 \times \frac{\sqrt{3}}{4}\left(L^{2}-3 A^{2}\right) \\
& =3 \sqrt{3}\left(1-(\sqrt{3}-r)^{2}\right) D_{\{111\}}^{2} .
\end{aligned}
$$

IUCr macros version 2.1.6: 2014/01/16 
The volume of the regular truncated octahedron $V_{\text {reg.t.oct. }}$ is obtained by considering the geometry of a regular octahedron of volume $V_{\text {reg.oct. }}$ with six pyramidal corners of volume $V_{\text {pyr. }}$ cut off:

$$
\begin{aligned}
V_{\text {reg.t.oct. }} & =V_{\text {reg.oct. }}-6 V_{\text {pyr. }} \\
& =1 / 2\left(\sqrt{3}-(\sqrt{3}-r)^{3}\right) D_{\{111\}}^{3} .
\end{aligned}
$$

\subsection{Thermodynamic Analysis for the Continuum Model}

We now calculate the change in energy per vacancy emitted as a function of the aspect ratio and the distance between $\{111\}$ facets, using the continuum approximation for the void volume. The change in energy as a function of the aspect ratio $r$, with $D_{\{111\}}$ constant, is

$$
\left(\frac{\partial E}{\partial r} \times \frac{\partial r}{\partial n}\right)_{D_{\{111\}}}=\frac{4\left(\sqrt{3} \gamma_{(111)}-\gamma_{(002)}\right) \Omega}{(\sqrt{3}-r) D_{\{111\}}} .
$$

The change in energy as a function of $D_{\{111\}}$, with $r$ constant, is

$$
\left(\frac{\partial E}{\partial D_{\{111\}}} \times \frac{\partial D_{\{111\}}}{\partial n}\right)_{r}=\frac{4\left(\sqrt{3} \gamma_{(111)}-\left(\sqrt{3} \gamma_{(111)}-\gamma_{(002)}\right)(\sqrt{3}-r)^{2}\right) \Omega}{\left(\sqrt{3}-(\sqrt{3}-r)^{3}\right) D_{\{111\}}} .
$$

The curves for the two energy changes are plotted in Fig. 7 (see the main text) as a function of $r$. We can determine which energy change is larger at a given aspect ratio or $D_{\{111\}}$ by examining the quantity $M$ defined as

$$
\begin{aligned}
M & =\left(\frac{\partial E}{\partial r} \times \frac{\partial r}{\partial n}\right)_{D_{\{111\}}}-\left(\frac{\partial E}{\partial D_{\{111\}}} \times \frac{\partial D_{\{111\}}}{\partial n}\right)_{r} \\
& =\left(\gamma_{(111)} r-\gamma_{(002)}\right) B
\end{aligned}
$$

where the coefficient $B$ is

$$
B=\frac{4 \sqrt{3} \Omega}{(\sqrt{3}-r)\left(\sqrt{3}-(\sqrt{3}-r)^{3}\right) D_{\{111\}}} .
$$

$B$ is always positive within an aspect ratio range of $(\sqrt{3}-\sqrt[6]{3}, \sqrt{3})$.

IUCr macros version 2.1.6: 2014/01/16 


\subsection{Truncated Octahedron in the Quantised Model}

A truncated octahedron can be described by the distances from the geometrical centre between each set of parallel facets. The continuum model above is a special case that assumes equal distances between $\{002\}$ or $\{111\}$. In the quantised model, we describe the distance from the void centre to each individual facet (hkl) in terms of the equivalent number of atomic layers $I_{(h k l)}$. The distance $d_{h k l}$ (see Fig.5 in the main text and Fig. S6 in the Supplementary Material) is the sum of $I_{(h k l)}+I_{(\bar{h} \bar{k} \bar{l})}+1$ for a particular set of parallel facets. An arbitrary set of $I_{(h k l)}$ will define an arbitrarily truncated octahedron. The equations below are for a FCC lattice only.

2.3.1. Surface Area of an Arbitrarily Truncated Octahedron. The total surface area of all the $\{002\}$ facets is

$$
S_{\{002\}}=S_{(200)}+S_{(020)}+S_{(002)}+S_{(\overline{2} 00)}+S_{(0 \overline{2} 0)}+S_{(00 \overline{2})} .
$$

As shown in Fig. S9(a), we take (200) as one of the facets for illustration. Equations for other facets can be obtained with similar reasoning.

$$
S_{(200)}=A^{\prime} \times A^{\prime \prime}
$$

where $A^{\prime}$ and $A^{\prime \prime}$ are the side lengths of the (200) facet. They are defined by pairs of $\{111\}$ facets being truncated by (200) facets.

$$
\begin{aligned}
& A^{\prime}=\frac{a}{\sqrt{2}}\left(I_{(1 \overline{1} 1)}+I_{(11 \overline{1})}-I_{(200)}\right), \\
& A^{\prime \prime}=\frac{a}{\sqrt{2}}\left(I_{(111)}+I_{(1 \overline{1} \overline{1})}-I_{(200)}\right) .
\end{aligned}
$$

where $a$ is the lattice parameter of aluminium.

IUCr macros version 2.1.6: 2014/01/16 


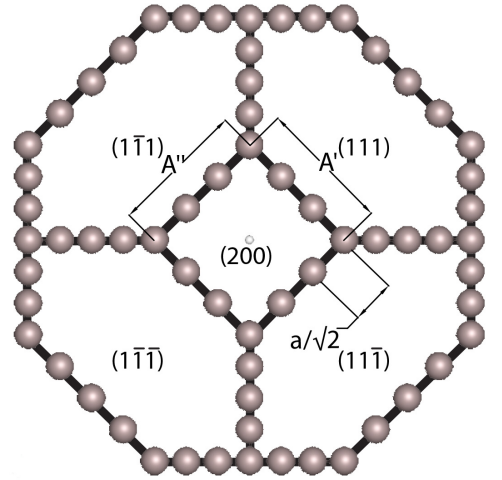

(a)

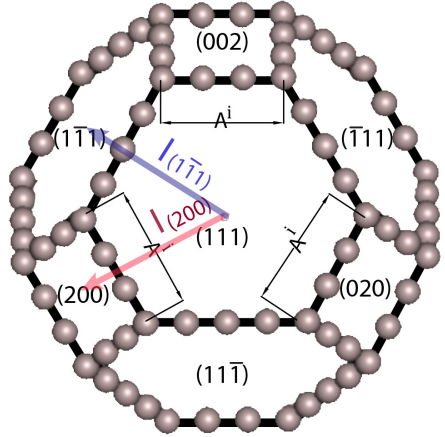

(b)

Fig. S9. (a) Schematic diagram of a void viewed in the [100] direction, showing the parameters used in the quantised model. The continuous line outlines the truncated octahedron with vacancies shown on the edges as grey spheres. All parameters are defined in the text. (b) Schematic diagram of a void viewed in the [111] direction. Arrows indicate $I_{(1 \overline{1} 1)}$ and $I_{(200)}$ respectively.

For the $\{111\}$ facets,

$$
S_{\{111\}}=S_{(111)}+S_{(\overline{1} 11)}+S_{(1 \overline{1} 1)}+S_{(11 \overline{1})}+S_{(\overline{1} \overline{1} 1)}+S_{(\overline{1} 1 \overline{1})}+S_{(1 \overline{1} \overline{1})}+S_{(\overline{1} \overline{1} \overline{1})} .
$$

This is shown in Fig. S9(b) for (111) as an example; other facets can be obtained using a similar reasoning.

$$
\begin{gathered}
S_{(111)}=\frac{\sqrt{3}}{4}\left(L_{(111)}^{2}-\sum\left(A^{i}\right)^{2}\right), \\
L_{(111)}=\frac{a}{\sqrt{2}}\left(I_{(11 \overline{1})}+I_{(1 \overline{1} 1)}+I_{(\overline{1} 11)}-I_{(111)}\right),
\end{gathered}
$$

where $L_{(111)}$ is the side-length of the octahedral (111) facets without truncation. $A^{i}$ corresponds to the side-length of each of the three $\{002\}$ facets intersecting (111) facets. 
2.3.2. Number of Vacancies in an Arbitrarily Truncated Octahedron. Now consider an arbitrarily truncated octahedron as the result of a cuboid with tetrahedral truncations at eight corners cut by $\{111\}$ facets. The total number of vacancies within the void, $n$, then is

$$
n=n_{0}-n_{1}+n_{2},
$$

where $n_{0}, n_{1}, n_{2}$ is the number of vacancies within the cuboid, the tetrahedral truncations and overlap areas of the tetrahedral truncations, respectively.

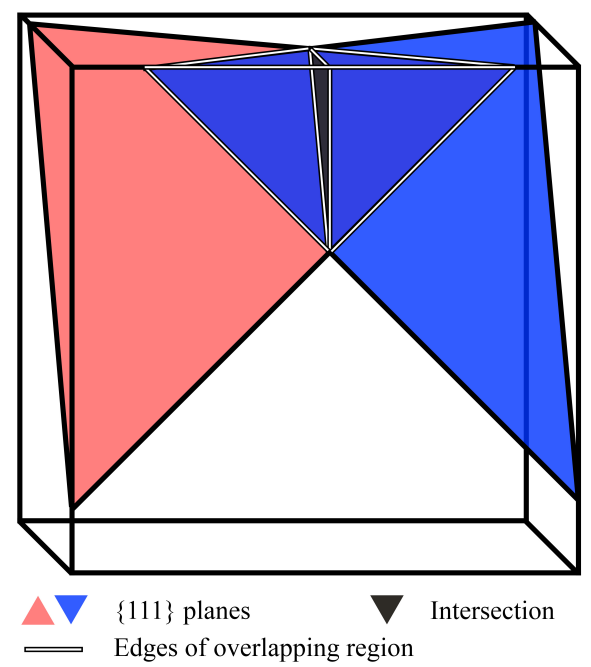

Fig. S10. Schematic diagram illustrating the algorithm for calculating the number of vacancies in an arbitrarily truncated octahedron. The cuboid is now truncated by two adjacent $\{111\}$ facets. The number of vacancies left is the total number of vacancies within the cuboid subtracted by the number of vacancies within the tetrahedral truncations plus the overlapping region between those tetrahedra. The overlapping region is composed of the intersection between two equivalent tetrahedra.

The number of vacancies, $n_{0}$, within the cuboid is

$$
n_{0}=\left\lfloor\left(I_{(200)}+I_{(\overline{2} 00)}+1\right)\left(I_{(020)}+I_{(0 \overline{2} 0)}+1\right)\left(I_{(002)}+I_{(00 \overline{2})}+1\right) / 2\right\rfloor+o,
$$


where

$$
o= \begin{cases}1 \quad & \text { if all } I_{\{002\}} \text { are even, } \\ & \text { or any two pairs of } I_{\{002\}} \text { of opposite facets are odd, } \\ 0 \quad & \text { otherwise. }\end{cases}
$$

The number of vacancies, $n_{1}$, within eight tetrahedral truncations by $\{111\}$ facets is

$$
n_{1}=\sum g\left(I_{\{111\}}, I_{\{002\}}\right) .
$$

Consider a (111) facet as an example. The number of vacancies within the tetrahedron bounded by (111), (200), (020) and (002) is

$$
\begin{aligned}
& g\left(I_{(111)}, I_{(200)}, I_{(020)}, I_{(002)}\right)= \\
& \begin{cases}\frac{p(p+1)(4 p-1)}{6} & \text { if } I_{(200)}+I_{(020)}+I_{(002)} \text { is even, } \\
\frac{p(p+1)(4 p+5)}{6} & \text { if } I_{(200)}+I_{(020)}+I_{(002)} \text { is odd, }\end{cases}
\end{aligned}
$$

where

$$
p=\left\lfloor\frac{I_{(200)}+I_{(020)}+I_{(002)}}{2}\right\rfloor-I_{(111)} .
$$

The number of vacancies within the fourteen overlapping areas, $n_{2}$, is

$$
n_{2}=\sum h\left(I_{\{111\}}, I_{\{002\}}\right) .
$$

where the function $h$ is defined below. Take the overlapping region of the tetrahedron cut off by (111) and (111) for example: they share the adjacent (002) and (020) facets. $h\left(I_{(111)}, I_{(\overline{1} 11)}, I_{(002)}, I_{(020)}\right)=$

$$
\begin{aligned}
& g\left(I_{(111)}, I_{(200)}, I_{(020)}, I_{(111)}-I_{(\overline{1} 11)}-1\right) \\
& +g\left(I_{(\overline{1} 11)}, I_{(200)}, I_{(020)}, I_{(\overline{1} 11)}-I_{(111)}-1\right)+q,
\end{aligned}
$$

where

$$
q= \begin{cases}\left(v^{2}+2 v\right) / 4 & \text { if } \mathrm{v} \text { is even } \\ \left(v^{2}+2 v+1\right) / 4 & \text { if } \mathrm{v} \text { is odd }\end{cases}
$$

and

$$
v=I_{(002)}+I_{(020)}-I_{(111)}-I_{(\overline{1} 11)}-1 .
$$




\section{Derivation of Vacancy Emission Rate}

In general, the vacancy emission rate $\frac{d n}{d t}$ is given by

$$
\frac{d n}{d t}=D_{v} \nabla C S
$$

where $D_{v}$ is the vacancy diffusivity, $\nabla C$ is the vacancy concentration gradient at the void surface and $S$ is the activated surface area from which vacancies are emitted (Crank, 1979). Assuming a regular truncated octahedron, only the $\{002\}$ facets are activated in Stage I, while both the $\{111\}$ and the $\{002\}$ facets are available for vacancy emission in Stage II.

We assume that the vacancy concentration reaches a steady state at the void surface under annealing. Consequently, the vacancy concentration profile satisfies the Laplace equation according to Fick's Second Law:

$$
\frac{\partial C}{\partial t}=D_{v} \nabla^{2} C=0
$$

At a given temperature $T$, the vacancy concentration $C_{v}$ at the void surface is given by:

$$
C_{v} / C_{0}=\exp \left\{(d E / d n) / k_{B} T\right\}
$$

where $C_{0}$ is the equilibrium vacancy concentration, $d E / d n$ is the change in void energy per vacancy emitted (see Eqs. S9 and S10) and $k_{B}$ is Boltzmann's constant.

With boundary conditions given by $C=C_{v}$ at the void surface and $C=C_{0}$ as the equilibrium vacancy concentration in the matrix far from the void, the equation for the vacancy concentration profile in the $\langle\mathrm{hkl}>$ direction is

$$
C(x)=C_{0}+\frac{0.5 D_{\{h k l\}}}{x}\left(C_{v}-C_{0}\right)
$$

where $D_{\{h k l\}}$ represents the distance between major facets $\{$ hkl $\}$ (either $\{002\}$ or $\{111\})$ and $x$ is the distance from the truncated octahedron centre along the $<$ hkl $>$

IUCr macros version 2.1.6: 2014/01/16 
directions (Crank, 1979). By assuming that each facet along a $<$ hkl $>$ direction is not flat but curved with constant curvature, we obtain a simple analytical solution to the Laplace equation, as written in Eq. S33.

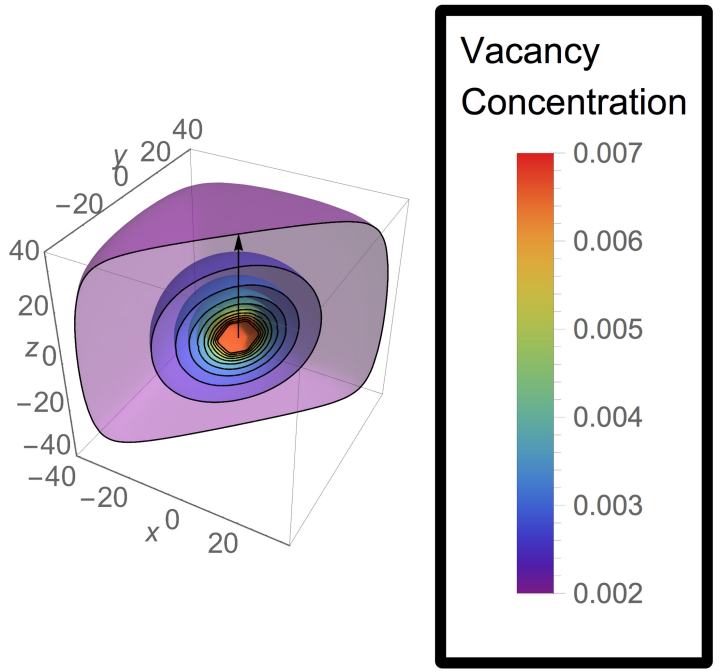

(a)
)

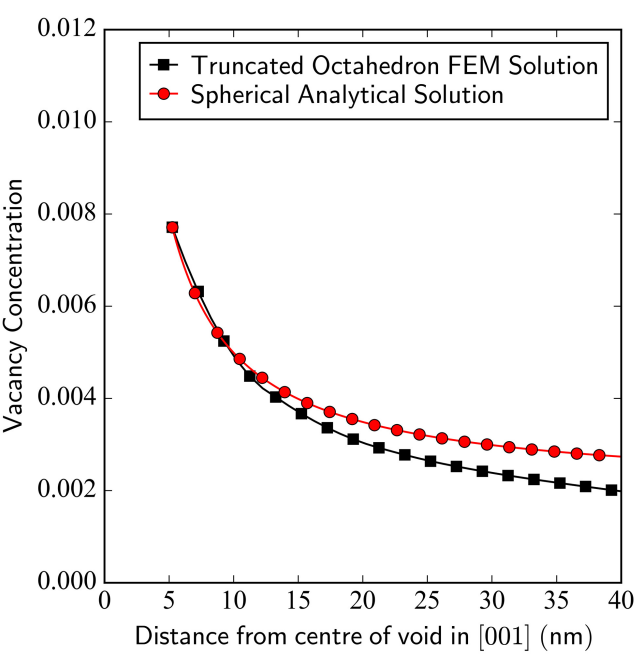

(b)

Fig. S11. (a) Calculation of the vacancy concentration profile around a truncated octahedral void with a distance between $\{111\}$ facets of $10 \mathrm{~nm}$ and an aspect ratio of 1.05 . The present case is for a temperature of $100^{\circ} \mathrm{C}$. The calculation was performed using the finite element method in Mathematica, with boundary conditions of concentration $C_{v}$ at the void surface and $C_{0}$ at the bounding box edges. The box edges are $80 \mathrm{~nm}$ in length in this case, which is the typical thickness of the void-containing regions examined by TEM. A vacancy formation energy of $0.2 \mathrm{eV}$ was chosen in order to reflect the experimental condition under continuous irradiation by the electron beam. The arrow indicates the $<001>$ direction. (b) Vacancy concentration as a function of distance from the centre of the void along $<001>$.

The numerical Laplace solution for a truncated octahedral geometry is calculated by the Finite Element Method (FEM) using Mathematica (see Fig. S11). Whereas in the analytical solution the vacancy concentration is assumed to reach its equilibrium value at infinity, FEM sets the equilibrium concentration $C_{0}$ at the box boundaries 
and $C_{v}$ at the void surface. This results in a difference in concentration between the two solutions away from the void surface, as shown in Fig. S11. However this difference will be reduced when larger FEM boundaries are considered. In any case, the vacancy concentration profile along a $<100>$ direction obtained numerically agrees well with the analytical solution in Eq. S33 and yields almost the same concentration gradient at the void surface. This good agreement justifies the use of the spherical assumption for the purpose of simple and reliable vacancy emission analysis, as shown below.

Taking the derivative of Eq. S33 with respect to distance, the concentration gradient at the void surface $\nabla C$ in the $<\mathrm{hkl}>$ direction is

$$
\nabla C=-\frac{2\left(C_{v}-C_{0}\right)}{D_{\{h k l\}}} .
$$

The self-diffusion coefficient $D_{s}$ is defined as

$$
D_{s}=\xi D_{v} C_{0} \Omega
$$

where $\xi$ is the correlation factor for self diffusion. For FCC structures, $\xi=0.781$ (Volin \& Balluffi, 1968). We can transform Eq. S30 into

$$
\frac{d n}{d t}=\frac{2 S D_{s}}{\xi D_{\{h k l\}} \Omega}\left(\exp \left\{(d E / d n) / k_{B} T\right\}-1\right)
$$

As the activated surface area for vacancy emission, $S$, and the energy reduction per vacancy emitted $d E / d n$ in Eq. S36 are different for the two stages of shrinkage, the model derived here differs from previous spherical models (Volin \& Balluffi, 1968; Westmacott et al., 1968). Thus, by combining Eqs. S6, S9 and S36, we can get the vacancy emission rate for Stage I of void evolution as plotted in Fig. 11(a) in the main text:

$$
\frac{d n}{d t}=-A_{1}\left(\exp \left(\frac{A_{2}}{\sqrt{3}-r}\right)-1\right) \frac{(\sqrt{3}-r)^{2}}{r}
$$

where

$$
A_{1}=\frac{6 D_{s} D_{\{111\}}}{\xi \Omega}
$$

IUCr macros version 2.1.6: 2014/01/16 
and

$$
A_{2}=\frac{4\left(\sqrt{3} \gamma_{(111)}-\gamma_{(002)}\right) \Omega}{k_{B} T D_{\{111\}}} .
$$

Again, here we assume a regular truncated octahedron. Similarly, combining Eqs. S6, S7, S10 and S36 leads to the vacancy emission rate for Stage II as plotted in the main text Fig.11(b):

$$
\frac{d n}{d t}=-A_{3}\left(\exp \left(\frac{A_{4}}{D_{\{111\}}}\right)-1\right) D_{\{111\}}
$$

where

$$
A_{3}=\frac{6 D_{s}\left((7-\sqrt{3}) r^{2}-4 \sqrt{3} r+3\right)}{r \xi \Omega}
$$

and

$$
A_{4}=\frac{4\left(\sqrt{3} \gamma_{(111)}-\left(\sqrt{3} \gamma_{(111)}-\gamma_{(002)}\right)(\sqrt{3}-r)^{2}\right) \Omega}{k_{B} T\left(\sqrt{3}-(\sqrt{3}-r)^{3}\right)} .
$$

$A_{1}, A_{2}, A_{3}$ and $A_{4}$ are all factors that include constant parameters.

\section{References}

Crank, J. (1979). The Mathematics of Diffusion. Oxford science publications. Clarendon Press. Volin, T. E. \& Balluffi, R. W. (1968). Physica Status Solidi (b), 25(1), 163-173.

Westmacott, K. H., Smallman, R. E. \& Dobson, P. S. (1968). Metal Science, 2(1), 177-181. 